rheuma plus $2020 \cdot 19: 224-229$ https://doi.org/10.1007/s12688-020-00383-x Online publiziert: 9 . Oktober 2020 (c) Der/die Autor(en) 2020

\section{Josef Hermann}

Universitätsklinik für Innere Medizin, Klinische Abteilung für Rheumatologie und Immunologie

Medizinische Universität Graz, Graz, Österreich

\title{
Algorithmus des Vorgehens beim unklaren Gelenkschmerz
}

Muskuloskeletale Schmerzen gehören zu den häufigsten Symptomen in der Medizin [8]. Unter Gelenkschmerzen werden häufig alle Schmerzen, die im Bereich eines Gelenks auftreten, subsummiert. Definitionsgemäß gehen Gelenkschmerzen jedoch von der Synovialmembran aus (Arthralgien) und entstehen durch eine Reizung von Nozizeptoren. Gelenkschmerzen müssen von Schmerzen, die außerhalb der Gelenkmembran im gelenknahen Knochen, in den Bändern oder in den Sehnenansätzen entstehen (Periarthralgien) abgegrenzt werden.

Im nachfolgenden Artikel wird auf die Definition entsprechender Gelenkschmerzen an den Extremitäten eingegangen, obwohl wir auch am Achsenskelett an den Intervertebral-, Costovertebral-, und Costotransversalgelenken Gelenkschmerzen vorfinden. In der Literatur finden sich nur wenige Beiträge meist aus dem Bereich der Bildgebung -, die sich mit den diagnostischen Pfaden zur Abklärung von Gelenkschmerzen beschäftigen, sodass der Inhalt dieses Artikels von der Expertise des Autors geprägt ist.

Um bei Patientinnen und Patienten mit Schmerzen am Bewegungsapparat differenzialdiagnostische Überlegungen anstellen zu können, müssen vorab die Fragen nach einem entzündlichen, nichtentzündlichen oder neuropathischen Schmerz und nach dem Ursprungsort des Schmerzes geklärt werden. Nur bei einem entzündlichen Gelenk- oder Sehnenscheidenschmerz sowie Schmerzen an Enthesen denkt man an eine entzündlich-rheumatische Erkrankung.

\section{Entzündlicher und nicht- entzündlicher Gelenkschmerz}

Suchen Patientinnen und Patienten mit einem Gelenkschmerz ärztlichen Rat, ist es in einem ersten Schritt erforderlich, zwischen einem nichtentzündlichen und einem entzündlichen Schmerz zu unterscheiden. Die Differenzierung dieser zwei Schmerzformen hat weitreichende diagnostische Konsequenzen, da ein entzündlicher Gelenkschmerz (Arthritis) auf eine entzündliche Erkrankung und damit möglicherweise rheumatische Erkrankung hinweist. Ein nichtentzündlicher Gelenkschmerz deutet hingegen auf funktionelle oder degenerative Veränderungen in einem Gelenk hin. Die Differenzierung zwischen einem entzündlichen und nichtentzündlichen Gelenkschmerz beeinflusst zudem die primären therapeutischen Maßnahmen, da ein entzündlicher Gelenkschmerz mit Antiphlogistika und ein nichtentzündlicher Gelenkschmerz mit Analgetika behandelt werden sollte.

Zur Unterscheidung zwischen einem entzündlichen und nichtentzündlichen Gelenkschmerz stehen uns vier diagnostische Methoden zur Verfügung: 1) die Schmerzanamnese, 2) die Bildgebung in Form des Ultraschalls und der Magnetresonanztomographie (MRT), 3) die laborchemischen Entzündungsparameter und 4) die invasiven Maßnahmen der Gelenkpunktion mit Synoviaanalyse und die Biopsie von schmerzhaften Strukturen.

\section{Schmerzanamnese}

Zur Unterscheidung zwischen einem entzündlichen und nichtentzündlichen Schmerz steht uns die Schmerzanamne- se als einfache, geräteunabhängige, aber zeitaufwändige Methode zur Verfügung. Die Schmerzanamnese steht am Anfang des diagnostischen Pfades, da sie die Prätestwahrscheinlichkeit für weiterführende technische Untersuchungen bestimmt [1]. Das Vorliegen eines Nachtschmerzes, eines Dauerschmerzes, eines Ruheschmerzes, einer Besserung des Schmerzes auf Kältezufuhr oder einer Morgensteifigkeit, die mehr als eine Stunde beträgt, deutet auf einen entzündlichen Gelenkschmerz hin [2].

Ein Nachtschmerz liegt dann vor, wenn Patienten durch den Gelenkschmerz in der Nacht aufgeweckt werden. Wenn der Patient lediglich in der Nacht auf dem Weg zur Toilette einen Gelenkschmerz verspürt, sollte nicht von einem Nachtschmerz gesprochen werden. Ein morgendlicher, bereits vor dem Aufstehen vorhandener Schmerz deutet ebenfalls auf einen entzündlichen $\mathrm{Ge}$ lenkschmerz hin und kann als mitigierter Nachtschmerz, der nicht zum Erwachen geführt hat, interpretiert werden.

Von einem Dauerschmerz spricht man, wenn der Gelenkschmerz während der gesamten Zeit des Wachseins unabhängig von körperlicher Tätigkeit vorhanden ist. Eine wechselnde Intensität des Gelenkschmerzes hat dabei keine diagnostische Bedeutung.

Von einem Ruheschmerz spricht man, wenn der Patient auch dann einen Gelenkschmerz verspürt, wenn sich die betroffene Gelenkregion unbelastet in einer Ruheposition befindet. Die Überprüfung des Ruheschmerzes in einem Gelenk gelingt am besten, wenn sich die Anamnese auf eine entspannte Ruhelage der schmerzhaften Region im Bett bezieht 


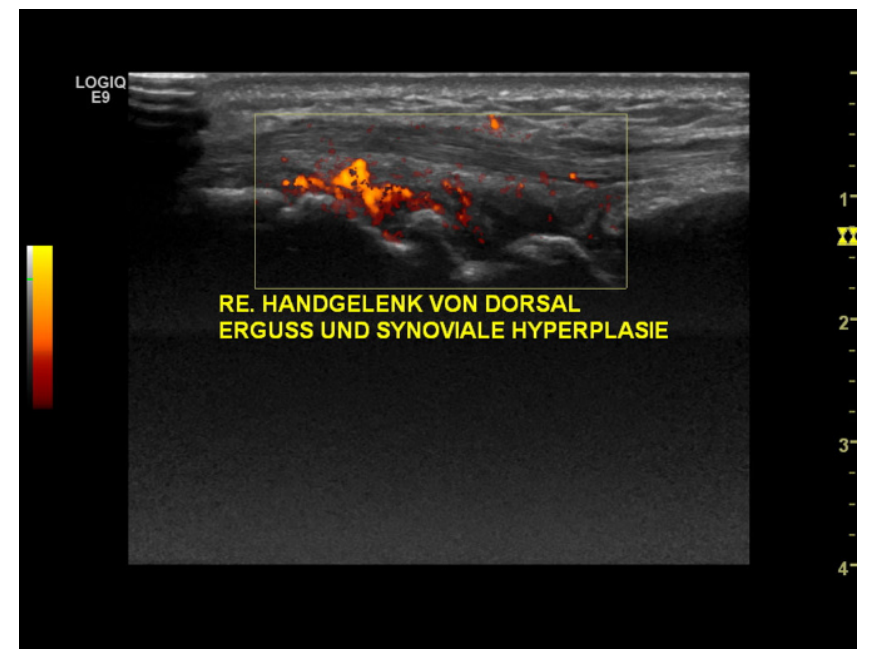

Abb. 1 ॥ Sonographische Darstellung eines rechten Handgelenks von dorsal im Längsschnitt. Es findet sich eine hyperplastische, echoarme Synovialis im Bereich des proximalen und distalen Handgelenks mit Hypervaskularität III ${ }^{\circ}$ rote Einfärbung) in der Power-Doppler-Sonographie. Dargestellt ist auch die Sehnenscheide der Musculus-extensor-digitorum-Sehne mit echoarmer hyperplastischer Synovialis und Hypervaskularität $I^{\circ}$

und keine ungewöhnliche Belastung der untersuchten Region vorausgegangen ist.

Patienten mit entzündlichen Gelenkschmerzen geben eine Besserung des Schmerzes bei lokaler Kältezufuhr an. Dabei ist allerdings zu beachten, dass kein zusätzlicher neuropathischer Schmerz vorliegt, die Kälteanwendung nur zu einer Kühlung der Hauttemperatur führt und das schmerzhafte Gelenk möglichst oberflächennah liegt, um mit der Kälteanwendung in Kontakt zu kommen.

Die Morgensteifigkeit ist ein selten erhebbares, aber insbesondere bei der rheumatoiden Arthritis vorhandenes Symptom, das auf eine Gelenkentzündung hindeutet, wenn sie mehr als $30 \mathrm{~min}$ andauert [19]. Die Morgensteifigkeit stellt eine schmerzunabhängige Bewegungseinschränkung dar, die von einer Functio laesa abgegrenzt werden muss und eine schmerzbedingte Bewegungseinschränkung beschreibt.

\section{Funktionell-rheumatologische Untersuchung}

Die funktionell-rheumatologische Untersuchung sollte an die Schmerzanamnese angeschlossen werden, um die Strukturen zu identifizieren, von denen der Schmerz ausgeht. Sie beinhaltet neben der Inspektion die aktive und passive Bewegungsprüfung, die Durchführung von Muskelprovokationstests sowie die Palpation der Strukturen der betroffenen Region.

Bei der Inspektion lassen sich neben Verfärbungen der Haut auch Schwellungen, Verdickungen und Deformierungen als möglicher Hinweis auf eine pathologisch veränderte Region erkennen.

Mit der aktiven und passiven Bewegungsprüfung können schmerzhafte artikuläre und periartikuläre Strukturen voneinander abgegrenzt werden. Führt die passive Untersuchung zu einer Schmerzauslösung oder Schmerzverstärkung, liegt ein artikuläres Geschehen vor. Ist lediglich die aktive - nicht aber die passive - Untersuchung mit einer Schmerzprovokation verbunden, kann von einem periartikulären Geschehen ausgegangen werden.

Muskelprovokationstests sind ein wichtiges Hilfsmittel zur weiteren Differenzierung von extraartikulär gelegenen schmerzhaften Strukturen. Bei entsprechenden anatomischen Kenntnissen und Vertrautheit mit den Muskelfunktionen können schmerzhafte Strukturen Sehnen, Sehnenscheiden und Enthesen zugeordnet werden.

Die funktionell-rheumatologische Untersuchung wird mit der Palpation der betroffenen Region abgeschlossen. Unter Einbeziehung einer Kontrollpalpation des umgebenden Gewebes und der Tiefenpalpation ist es möglich, die Ergebnisse der funktionellen Untersuchung zu bestätigen bzw. zu ergänzen und den Ursprungsort des Schmerzgeschehens zu fokussieren.

Ergibt sich aus der Schmerzanamnese und der funktionell-rheumatologischen Untersuchung eine ausreichend hohe Prätestwahrscheinlichkeit für ein entzündliches Geschehen in einem oder in mehreren Gelenken, können sonographische und MR-tomographische sowie laborchemische Untersuchungen angeschlossen werden, um die Posttestwahrscheinlichkeit für das Vorliegen einer Arthritis, Enthesitis oder Tendovaginitis und damit für eine entzündlichrheumatische Erkrankung zu erhöhen.

\section{Gelenksonographie}

Die Gelenksonographie wurde erstmals 1978 als Methode zur Verlaufsuntersuchung einer lokalen Yittrium-Therapie bei Patienten mit aktiver rheumatoider Arthritis verwendet [19]. Mit der technischen Möglichkeit, eine Power-DopplerUltraschalluntersuchung durchführen zu können, stieg ab 2001 das Interesse der Rheumatologen am Einsatz dieser Untersuchungstechnik merklich an [7, 11]. Mit der Ultraschalltechnik unter Verwendung der Power-Doppler-Sonographie lassen sich indirekte Hinweise auf eine Arthritis, Tendovaginitis oder Enthesitis finden [4]. Dazu gehören der Nachweis vermehrter Flüssigkeit, der Nachweis einer hypoechogenen, verdickten Synovialmembran oder Sehnenscheide und die Darstellung einer Hypervaskularität in den pathologisch

Hier steht eine Anzeige.

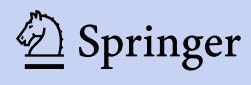


veränderten Strukturen ([9]; • Abb. 1). Sonographisch lassen sich auch strukturelle Veränderungen wie Erosionen, Destruktionen oder Osteoproliferationen als Hinweis auf einen stattgehabten entzündlich-rheumatischen Prozess finden. Die Sonographie ist allerdings stark untersucherabhängig und so sensitiv und auflösungsstark, dass die Gefahr einer Überinterpretation von Befunden besteht. Die Aussagekraft des PowerDoppler-Signals ist wiederum von der Lokalisation des $\mathrm{zu}$ untersuchenden Gewebes in der Tiefe, von der Geräteeinstellung und vom Gerätetyp abhängig [18].

\section{Magnetresonanztomographie}

Die MRT ist ebenso wie die Sonographie in der Lage, indirekte Hinweise auf entzündliche Veränderungen in Gelenken, Sehnenscheiden und Enthesen zu liefern [12]. Durch die Verwendung von T2-gerichteten Sequenzen können Flüssigkeitsansammlungen und durch die Verwendung von Gadolinium-haltigem Kontrastmittel (Gadopentetat-Dimeglumin) eine Hypervaskularität als Hinweis auf ein entzündliches Geschehen dokumentiert werden. In klinischen Studien findet sich mit über $80 \%$ eine gute Übereinstimmung zwischen MR-tomographischen und klinischen Hinweisen auf eine Entzündung, wobei klinisch als Arthritiden imponierende Veränderungen MR-tomographisch sowohl Synovitiden als auch Tendovaginitiden darstellen können [10]. Ein maßgeblicher Vorteil der MRT gegenüber der Sonographie besteht in der Detektion von Knochenödemen als Hinweis auf eine Knochen(mark)entzündung (Ostitis), in der Detektion von Periostitiden und in der Darstellungsmöglichkeit von tiefliegenden Strukturen besonders im Hüftoder Schultergürtelbereich [17]. MRtomographisch können zudem morphologische Veränderungen wie Erosionen, Knorpelverluste und Sehnenrupturen detektiert werden [3]. Sonographie und MRT sind somit verwandte Methoden in der Darstellung von entzündlichen Gewebsveränderungen. Die MRT erlaubt zudem die Evaluation von sonogra-

rheuma plus $2020 \cdot 19: 224-229$ https://doi.org/10.1007/s12688-020-00383-x

(c) Der/die Autor(en) 2020

\section{J. Hermann \\ Algorithmus des Vorgehens beim unklaren Gelenkschmerz}

\section{Zusammenfassung}

Gelenkschmerzen gehören zu den häufigsten Symptomen in der Medizin. Um Gelenkschmerzen diagnostisch einordnen zu können, ist eine Kategorisierung des Schmerzes in einen entzündlichen, nichtentzündlichen oder neuropathischen Schmerz hilfreich. Zudem muss der Schmerz einer anatomischen Struktur zugeordnet werden, da nur bei einem Schmerz, der von der Synovialmembran ausgeht, von einem Gelenkschmerz gesprochen werden kann. Für die Einordnung des Schmerzes in einen entzündlichen oder nichtentzündlichen Schmerz stehen uns methodisch die Schmerzanamnese, als bildgebende Methoden der Ultraschall und die Magnetresonanztomo- graphie und die Entzündungsparameter zur Verfügung. In unklaren Situationen und zur Sicherung eines entzündlichen Geschehens sind eine Synoviaanalyse und eventuell die Biopsie erforderlich. Um den Ort des Schmerzgeschehens zu lokalisieren, können wir die funktionelle Untersuchung, die Sonographie, die Magnetresonanztomographie und eventuell die Röntgenuntersuchung heranziehen.

\section{Schlüsselwörter}

Schmerzanamnese - Entzündlich .

Muskuloskeletaler Ultraschall · Magnetresonanztomographie (MRT) · Synoviaanalyse

\section{Algorithm for the Work-up of Ambiguous Joint Pain}

\section{Abstract}

Joint pain is one of the most frequent symptoms in medicine. In the clinical workup, one should try to classify joint pain as inflammatory, non-inflammatory and neuropathic. In addition, the pain should be assigned to a specific anatomical structure because only pain evolving from the synovial membrane can be categorized as joint pain. To differentiate joint pain into inflammatory or non-inflammatory pain anamnesis, ultrasound, magnetic resonance imaging and inflammatory markers are applied. To make

phisch unzugänglichen Strukturen und von intraossären Veränderungen.

\section{Entzündungsparameter}

In der klinischen Praxis werden zur Entscheidung, ob ein entzündliches oder nichtentzündliches Geschehen vorliegt, regelhaft die laborchemischen Entzündungsparameter C-reaktives Protein und seltener die Blutsenkungsgeschwindigkeit herangezogen. Vor dem unreflektierten Umgang mit Entzündungsparametern bei Patienten mit Gelenkschmerzen sei an dieser Stelle jedoch gewarnt. Gerade bei entzündlich-rheumatischen Erkrankungen besteht eine geringe Korrelation zwischen den laborchemischen a definite diagnosis of inflammatory joint pain synovial fluid analysis and occasionally biopsy is necessary. To localize the painful structure functional examination, followed by ultrasonography, magnetic resonance imaging or occasionally radiography are useful methods in clinical practice.

\section{Keywords}

Pain history · Inflammatory · Musculoskeletal ultrasonography · Magnetic resonance imaging (MRI) - Synovial fluid analysis 
Hier steht eine Anzeige.

\section{曾 Springer}




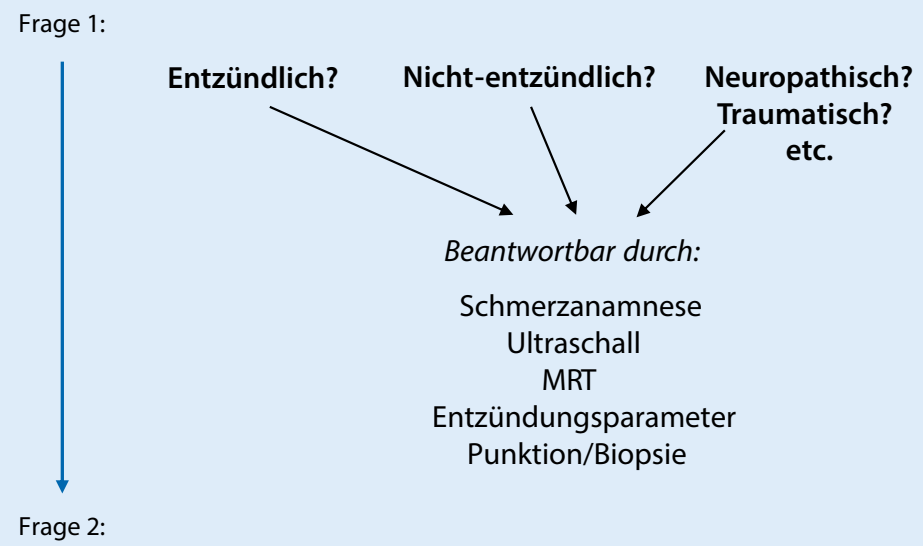

Betroffene Struktur (Gelenk, Sehne/Sehnenscheide,Enthesis, Bursa?)

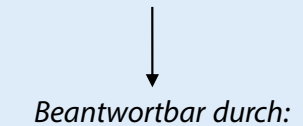

Funktionelle Untersuchung Ultraschall

MRT

Röntgen/CT

Frage 3:



Rheumatische Erkrankung?

Abb. 2 ^ Algorithmus zur Abklärung von unklaren Gelenkschmerzen aus rheumatologischer Sicht. MRT Magnetresonanztomographie

zwischen einem entzündeten oder nichtentzündeten Gelenkpunktat nicht angegeben werden [15]. Bei einer Leukozytenzahl bis zu 100/ $\mu$ l besteht sicher keine Gelenkinflammation, wobei die Leukozytenzahl jedoch invers mit der Größe des Gelenks zu korrelieren scheint [14, 16]. Bis zu einer Leukozytenzahl von 500/ $\mu$ l scheinen keine klinischen Zeichen einer Arthritis vorzuliegen [13]. Allgemein wird akzeptiert, dass ab einer Leukozytenzahl von 2000/ $\mu$ l eine Arthritis besteht [6].

\section{Lokalisation des Gelenk- schmerzes}

Stellen sich Patientinnen und Patienten mit einem Gelenkschmerz vor und wurde dieser als entzündlich oder nichtentzündlich kategorisiert, muss der Schmerz der tatsächlich erkrankten Struktur zugeordnet werden. Nur wenn ein entzündlicher Schmerz von der Synovialmembran ausgeht, kann von einer Arthritis gesprochen werden.

Um die schmerzhafte Struktur zu finden, stehen folgende Methoden zur Verfügung: 1. funktionelle Untersuchung (aktive und passive Bewegungsprüfung, Muskelprovokationstests, Palpation),
2. Bildgebung (Ultraschall, MRT, Röntgen).

\section{Funktionelle Untersuchung}

Die funktionelle Untersuchung beginnt mit der aktiven Bewegungsprüfung. Dabei werden die Patientinnen und $\mathrm{Pa}$ tienten aufgefordert, die Bewegungen entsprechend der im jeweiligen Gelenk bestehenden Freiheitsgrade auszuführen. Ist die aktive Bewegungsprüfung schmerzhaft oder mit einer Zunahme von bestehenden Schmerzen verbunden, wird die passive - vom Untersucher ausgeführte - Untersuchung der schmerzhaften Region angeschlossen. Ist die passive Untersuchung mit einer Schmerzauslösung oder Schmerzverstärkung verbunden, bestehen Hinweise auf eine schmerzhafte Gelenkstruktur. Ist die passive Untersuchung im Gegensatz zur aktiven Bewegungsprüfung frei, liegt ein periartikuläres Geschehen vor. In diesem Fall führen Muskelprovokationstests und die Palpation zum Auffinden der betroffenen Strukturen (Sehnen, Bursen, Enthesen etc.). Mit der funktionellen Untersuchung können gleichzeitig auch Funktionsdefizite gefunden und quantifiziert werden.

\section{Bildgebung zum Auffinden schmerzhafter Strukturen}

Die Bildgebung soll an die funktionelle Untersuchung angeschlossen werden, um eine möglichst hohe Prätestwahrscheinlichkeit für die in der angeschlossenen Bildgebung gefundenen pathologisch veränderten Strukturen zu erzeugen. Nur bei einer entsprechend hohen Prätestwahrscheinlichkeit kann eine in der Bildgebung auffällige pathologische Struktur den Beschwerden des Patienten mit hoher Sicherheit zugeordnet werden.

An den Extremitäten steht für die überwiegende Anzahl der Fragestellungen die Small-Part-Sonographie als strahlungsfreie und leicht verfügbare Untersuchungsmethode zur Verfügung. Falls die Detektion einer pathologischen Struktur in sonographisch schwer zugänglichen Regionen wie im Hüft- oder Schulterbereich notwendig ist, und zum Nachweis einer intraossär gelegenen 
pathologischen Struktur ist die Durchführung einer MRT erforderlich. Strukturelle Knochenveränderungen können weiterhin anhand von radiographischen Untersuchungen am besten identifiziert werden.

\section{Fazit}

Werden Patientinnen und Patienten mit einem Gelenkschmerz beim Arzt vorstellig, sollte als erste diagnostische Maßnahme eine gezielte Schmerzanamnese zur Differenzierung des Schmerzes in einen entzündlichen, nichtentzündlichen und neuropathischen Schmerz erfolgen (- Abb. 2). Anschließend sollen schmerzhafte Strukturen durch eine funktionelle Untersuchung der betroffenen Region lokalisiert werden. Ist die Schmerzanamnese hinsichtlich der $\mathrm{Zu}$ ordnung des Schmerzes nicht konklusiv und/oder lassen sich die schmerzhaften Strukturen durch die funktionelle Untersuchung nicht eindeutig zuordnen, sollte ein bildgebendes Verfahren im Fall einer Schmerzmanifestation an den Extremitäten in Form einer SmallPart-Sonographie - durchgeführt werden. Die Sonographie bietet weiters den Vorteil, einen möglichen Zugangsweg und die Erfolgsaussichten für eine diagnostische Gelenkpunktion abschätzen zu können. Lassen sich sonographisch Entzündungsgeschehen und pathologische Strukturen nicht eindeutig identifizieren, bzw. liegen diese intraossär, empfiehlt sich zur weiteren diagnostischen Abklärung an den Extremitäten die Durchführung einer MRT mit Gadoliniumdarstellung. Entzündungsparameter können zur Detektion eines entzündlichen Geschehens unterstützend angefordert werden, haben aber gerade bei rheumatologisch Erkrankten eine teilweise niedrige Sensitivität und Spezifität. Für den definitiven Nachweis einer entzündlichen Gelenksaffektion (Arthritis) ist eine Gelenkpunktion mit anschließender Synoviaanalyse und dem Nachweis von mehr als 2000 Leukozyten/ $\mu \mathrm{l}$ im Gelenkpunktat erforderlich.

Erst wenn anhand der angeführten Methoden die Zahl und Verteilung der Arthritiden sowie der Enthesitiden, Bursitiden und Tendovaginitiden erhoben werden, ist es möglich, differenzialdiagnostische Überlegungen hinsichtlich der zugrundeliegenden Gelenkerkrankung anzustellen und ein rheumatologisches oder orthopädisches Vorgehen einzuleiten.

\section{Korrespondenzadresse}

Priv.-Doz. Dr. Josef Hermann

Universitätsklinik für Innere Medizin, Klinische Abteilung für Rheumatologie und Immunologie, Medizinische Universität Graz Auenbruggerplatz 15, $8036 \mathrm{Graz}$, Österreich josef.hermann@medunigraz.at

Funding. Open access funding provided by Medical University of Graz.

\section{Einhaltung ethischer Richtlinien}

Interessenkonflikt. J. Hermann gibt an, dass kein Interessenkonflikt besteht.

Für diesen Beitrag wurden vom Autor keine Studien an Menschen oder Tieren durchgeführt. Für die aufgeführten Studien gelten die jeweils dort angegebenen ethischen Richtlinien.

Open Access. Dieser Artikel wird unter der Creative Commons Namensnennung 4.0 International Lizenz veröffentlicht, welche die Nutzung, Vervielfältigung Bearbeitung, Verbreitung und Wiedergabe in jeglichem Medium und Format erlaubt, sofern Sie den/die ursprünglichen Autor(en) und die Quelle ordnungsgemäßnennen, einen Link zur Creative Commons Lizenz beifügen und angeben, ob Änderungen vorgenommen wurden.

Die in diesem Artikel enthaltenen Bilder und sonstiges Drittmaterial unterliegen ebenfalls der genannten Creative Commons Lizenz, sofern sich aus der Abbildungslegende nichts anderes ergibt. Sofern das betreffende Material nicht unter der genannten Creative Commons Lizenz steht und die betreffende Handlung nicht nach gesetzlichen Vorschriften erlaubt ist, ist für die oben aufgeführten Weiterverwendungen des $\mathrm{Ma}$ terials die Einwilligung des jeweiligen Rechteinhabers einzuholen.

Weitere Details zur Lizenz entnehmen Sie bitte der Lizenzinformation auf http://creativecommons.org/ licenses/by/4.0/deed.de.

\section{Literatur}

1. Ali Y (2018) Rheumatologic tests: a primer for family physicians. Am Fam Physician 98:164-170

2. Bierma-Zeinstra SM, Bohnen AM, Verhaar JA et al (2000) Sonography for hip joint effusion in adults with hip pain. Ann Rheum Dis 59:178-182

3. Emery P, Van Der HeijdeD, Ostergaard Metal (2011) Exploratory analyses of the association of MRI with clinical, laboratory and radiographic findings in patients with rheumatoid arthritis. Ann Rheum Dis 70:2126-2130
4. Hassan S (2018) Overview of musculoskeletal ultrasound for the clinical rheumatologist. Clin Exp Rheumatol 36(Suppl 114):3-9

5. Jindal S, Kaushik R, Raghuvanshi S et al (2018) Gray scale and power doppler ultrasonographic findings in the assessment of disease activity and their correlation with disease activity parameters in rheumatoid arthritis. Curr Rheumatol Rev 14:153-162

6. Johnson MW (2000) Acute knee effusions: a systematic approach to diagnosis. Am Fam Physician 61:2391-2400

7. Joshua F, Edmonds J, Lassere M (2006) Power Doppler ultrasound in musculoskeletal disease: a systematic review. Semin Arthritis Rheum 36:99-108

8. Kohlmann T (2003) Musculoskeletal pain in the population. Schmerz 17:405-411

9. Koski JM, Saarakkala S, Helle M et al (2006) Power Doppler ultrasonography and synovitis: correlating ultrasound imaging with histopathological findings and evaluating the performance of ultrasound equipments. Ann Rheum Dis 65:1590-1595

10. Krabben A, Stomp W, Huizinga TW et al (2015) Concordance between inflammation at physical examination and on MRI in patients with early arthritis. Ann Rheum Dis 74:506-512

11. Magarelli N, Guglielmi G, Di Matteo L et al (2001) Diagnostic utility of an echo-contrast agent in patients with synovitis using power Doppler ultrasound: a preliminary study with comparison to contrast-enhanced MRI. Eur Radiol 11:1039-1046

12. Mcqueen FM (2009) The MRI view of synovitis and tenosynovitis in inflammatory arthritis: implications for diagnosis and management. Ann NYAcad Sci 1154:21-34

13. Pascual E (1991) Persistence of monosodium urate crystals and low-grade inflammation in the synovial fluid of patients with untreated gout. Arthritis Rheum 34:141-145

14. Pascual E (1989) Analysis of synovial fluid from healthy knees. Comparison with fluid from asymptomatic knees in RA, SLE and gout. $\mathrm{Br} J$ Rheumatol 31:219

15. Pascual E, Jovani V (2005) Synovial fluid analysis Best Pract Res Clin Rheumatol 19:371-386

16. Pascual-Gomez $E$ (1989) Joint size influence on the leucocyte count of inflammatory synovial fluids. $\mathrm{Br}$ JRheumatol 28:28-30

17. Soini I, Kotaniemi A, Kautiainen $\mathrm{H}$ et al (2003) US assessment of hip joint synovitis in rheumatic diseases. A comparison with MR imaging. Acta Radiol 44:72-78

18. Ten Cate DF, Luime JJ, Van Der Ven M et al (2013) Very different performance of the power Dopple modalities of several ultrasound machines ascertained by a microvessel flow phantom. Arthritis Res Ther 15:R162

19. Van Der Ven M, Van Der Veer-Meerkerk M, Ten Cate DF et al (2017) Absence of ultrasound inflammation in patients presenting with arthralgia rules out the development of arthritis. Arthritis Res Ther 19:202

Hinweis des Verlags. Der Verlag bleibt in Hinblick auf geografische Zuordnungen und Gebietsbezeichnungen in veröffentlichten Karten und Institutsadressen neutral. 